\title{
Damping Force Control of A Semi-active Suspension System Using Cuckoo Search Optimized PID Method
}

\author{
WONG Pak Kin ${ }^{1, a}$, MA Xinbo ${ }^{1, b^{*}}$, ZHAO Jing ${ }^{1, c}$, XIE Zhengchao ${ }^{1, d}$, \\ ZHAO Rongchen ${ }^{1, e}$ \\ ${ }^{1}$ Department of Electromechanical Engineering, University of Macau, Taipa, Macau \\ afstpkw@umac.mo, byb57467@umac.mo, cyb27437@umac.mo, dzxie@umac.mo, \\ eyb47437@umac.mo
}

Keywords: SAS system, Damping force control, CS-PID.

\begin{abstract}
In the automotive engineering, semi-active suspension (SAS) system has been one of the most attractive research projects. The SAS system usually refers to the damping force control using an adjustable damper. This research proposes a cuckoo search optimized proportionalintegral-derivative (CS-PID) strategy for the damping force control system in order to improve the vehicle performance under different driving conditions and to improve the handling stability and smoothness of driving. Firstly, a quarter car dynamic model with an air spring and an adjustable damper is developed. Subsequently, the CS-PID controller is designed to generate the desired damping force according to the vehicle states in a real-time. Simulation results reveal that the vehicle performance can be greatly improved with the proposed controller.
\end{abstract}

\section{Introduction}

Suspension systems in the vehicle are normally classified as passive suspension system, active suspension system and semi-active suspension (SAS) system. Considering the preponderances of controllability, low cost, less power consumption and simple construction, the studies on the SAS systems have been a popular trend in the vehicle dynamics to provide a good ride comfort for the driver [1].

The performance of the SAS system mainly depends on the control strategy. In the existing literature, various control strategies are available for the SAS system such as skyhook control [2], proportional-integral-derivative (PID) control [3], ground-hook control [4], and sliding mode control [5]. In conclusion, the control schemes of SAS are classified into three types. The first type is to achieve simple control by solving mechanical problems. The second type of strategy is developed according to the dynamics models and control theory of linear time-invariant (LTI) system. The third type of strategy is to develop a highly non-linear, strongly coupled and time-varying system for the reason that the linear system models are not accurate enough and precise mathematical models are hard to build. Even though these control strategies have been developed, the quick response, simple calculation and good utilization cannot be guaranteed in practice. Considering the wide utilization and good performance in response, the PID strategy is suitable for the SAS system control in practice.

Even though the PID algorithm has good performance in an industrial application, the determination of gains (e.g. gain P, gain I and gain D) is still a problem, especially for complex system. Taking this point a step further, modern optimization algorithm is considered. Cuckoo search, as one of the modern optimization techniques with more efficient randomization property (with the use of Levy flight) and fewer parameters (population size and discovery probability only), has been proved to achieve more robust performance than other famous techniques, such as genetic algorithms, particle swarm optimization and artificial bee colony algorithm in PID controller optimization [6, 7]. Therefore, a CS-based optimal PID (CS-PID) controller is developed for the SAS system.

Based on the above discussion, this paper proposes a CS-PID controller to achieve the ride 
comfort in the SAS system. The rest of this paper is organized as follows: Section 2 constructs the quarter car dynamic model. Section 3 presents the CS-PID controller. The simulation and analysis are illustrated in Section 4. Finally, conclusions are given in Section 5.

\section{Quarter Car Dynamic Model}

A quarter car model with two degrees of freedom, as presented in Fig. 1, is adopted in this research to analyze the proposed system. In order to get a clear perspective of the presented system for the controller design, the motions of the quarter car are evaluated.

In consideration of the vertical motion of sprung mass and unsprung mass, the dynamic equations of the suspension system are written as

$$
\left\{\begin{array}{l}
m_{s} \ddot{z}_{s}=-k_{s}\left(z_{s}-z_{u}\right)-c_{s}\left(\dot{z}_{s}-\dot{z}_{u}\right)-u(t) \\
m_{u} \ddot{z}_{u}=k_{s}\left(z_{s}-z_{u}\right)+c_{s}\left(\dot{z}_{s}-\dot{z}_{u}\right)-k_{t}\left(z_{u}-z_{r}\right)+u(t)
\end{array},\right.
$$

where, $m_{s}$ and $m_{u}$ denote the sprung mass and the unsprung mass, respectively; $k_{s}$ and $c_{s}$ are the stiffness and the damping coefficient of the passive suspension element; $k_{t}$ stands for the tire stiffness; $z_{s}, z_{u}$ and $z_{r}$ represent the displacements of the sprung mass, the unsprung mass and the road disturbance, respectively; and $u(t)$ is the control damping force (i.e. additional damping force).

Defining $x=\left[z_{s} \dot{z}_{s} z_{u} \dot{z}_{u}\right]^{T}$ as the state variables, the governing equation shown in Eq. 1 can be rewritten in the state-space form as

$$
\dot{x}(t)=A x(t)+B z_{r}+D u(t),
$$

where, $A$ is the state variable matrix; $B$ is the control input matrix; and $D$ is the road input matrix.

In the quarter car suspension model, the air spring used is a rolling lobe air spring, and its modeling details can be found in [8]. So, the modeling process is not presented here and the corresponding parameters used for the air spring modeling can be referred to a normal passenger vehicle. The parameters used for the modeling or the quarter car model are depicted in Table 1.

Table 1. Parameters of the quarter car suspension.

\begin{tabular}{ccc}
\hline Parameter & Notation & Value \\
\hline $\mathrm{c}_{\mathrm{s}}$ & damping coefficient (passive) & $1500[\mathrm{Nm} / \mathrm{s}]$ \\
$\mathrm{k}_{\mathrm{t}}$ & stiffness of the tire & $160000[\mathrm{~N} / \mathrm{m}]$ \\
$\mathrm{k}_{\mathrm{s}}$ & stiffness of air spring & variable \\
$\mathrm{m}_{\mathrm{s}}$ & sprung mass & $300[\mathrm{~kg}]$ \\
$\mathrm{m}_{\mathrm{u}}$ & unsprung mass & $25[\mathrm{~kg}]$ \\
\hline
\end{tabular}

\section{CS-PID Controller}

PID Controller. In this research, the vertical acceleration of the sprung mass is selected as the measured variable and its desired value is set as zero. The error is regarded as the control input which can be presented as

$$
e(t)=\ddot{z}_{s}-0=\ddot{z}_{s} .
$$

With the control input, the control output of the PID controller can be described as,

$$
u(t)=k_{p} e(t)+k_{i} \int e(t) d t+k_{d} \frac{d}{d t} e(t)
$$

where, $u(t)$ is the control damping force, and then it is delivered to the quarter car model as an additional damping force.

At this point, by subjecting a standard road excitation with some pits and bumps [9] (as shown in Fig. 2), the gains for PID controller can be adjusted via simulation with the parameters in Table 3. 
Through several numerical simulation, the gains of the PID controller are $k_{p}=998.42, k_{i}=2548.53$, $k_{d}=1012.56$.

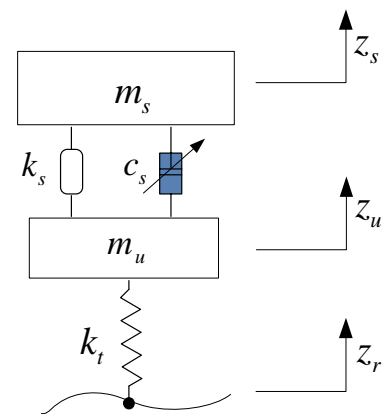

Fig. 1 Quarter car dynamic model

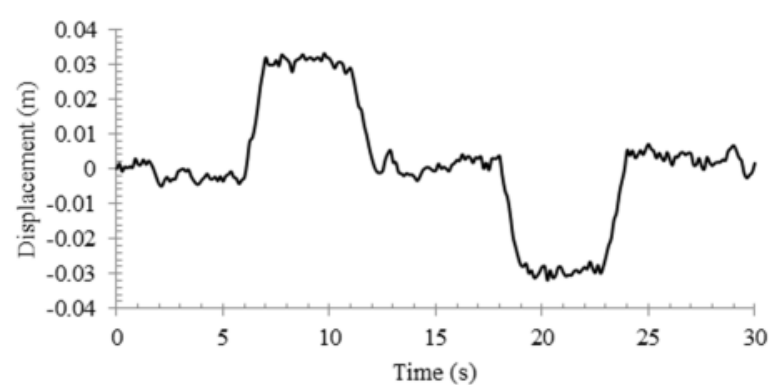

Fig. 2: Road Input of Quarter Car Suspension [9].

CS. The PID gains obtained through numerical simulation are used to find the nearby values of the PID gains. In order to find the optimum PID gains, the CS algorithm is proposed to further optimize the PID controller. The basic action of the CS algorithm is to get a new population $P^{\prime}$ with $P$ by carrying out three important rules: 1) Every cuckoo lays one egg only and is dumped in a arbitrarily chosen nest; 2) The best nests with eggs of high fitness take along the potential solution and are moved onto a next generation; 3) Number of available hosts is finite and a host can become aware of an alien egg with a probability that is from 0 to $1[6,7]$.

To generate a new nest for cuckoos, a Lévy flight is carried out by

$$
x_{i}(t+1)=x_{i}(t)+a \oplus \operatorname{Levy}(\lambda),
$$

where, $x_{i}(t+1)$ is the new solution for cuckoo $i$ and $x_{i}(t)$ is the current solution at the iteration $t ; a$ is the step size which is 1 in most cases; $\bigoplus$ is a product of entry-wise multiplications; $\lambda$ is a constant with the value of $1.5 ; \operatorname{Levy}(\lambda)$ is the Lévy flight which gives a random walk, and it can be expressed as

$$
\operatorname{Levy}(\lambda) \sim u=t^{-\lambda}(1 \leq \lambda \leq 3) .
$$

Eq. 7 can explain that the Lévy flight complies with the Lévy distribution with an limitless variance and limitless mean [6].

Determination of the PID gains using CS. Before CS optimization, the following parameters are selected for maximum accuracy and efficiency:

1. Number of generation $=500$

2. Number of nests $=15$

3. Probability perceiving alien egg $=0.25$.

With the initial gains of the PID controller, the following steps are conducted to determine the PID gains using CS:

(1) Initialization of a nest, a new nest candidate $x_{n}$ is generated randomly.

(2) By combing the initial PID gains, the road input cycle and the quarter car model, the fitness of each nest is evaluated so as to make the deflection of sprung mass acceleration as small as possible. The fitness function can be expressed as,

$$
F_{o b j}=\min \int_{0}^{\tau}\left(|e(t)|+|e(t)|^{2}+t|e(t)|\right) d t .
$$

where, $\tau$ is the length of the road input cycle and it is set as 30 seconds in this case study, $e(t)$ is the error as shown in Eq. 3.

(3) Comparing the fitness of the nest $x_{n}$ with the nest $x_{n-1}$. If the fitness of the nest $x_{n}$ is much bigger, the nest $x_{n}$ is replaced with the nest $x_{n-1}$. Otherwise, the nest $x_{n}$ will be taken to compare with the fitness of the best nest $x_{\text {best }}$. The $x_{\text {best }}$ is firstly set as the initial gains of the PID controller $\left(k_{p}=\right.$ 
998.42, $\left.k_{i}=2548.53, k_{d}=1012.56\right)$.

(4) If the fitness of the nest $x_{n}$ is much bigger as compared with the $x_{\text {best }}$, the best nest $x_{\text {best }}$ stays unchanged. Otherwise, the nest $x_{n}$ will be taken as the new solution, replacing the best nest $x_{\text {best }}$.

(5) New nest $x_{n}$ is generated from $x_{n-1}$ randomly using Levy flight in Eq. 6 in the next iteration.

(6) Abandon of some nests and generate new nests through crossover. The cycle is repeated again and again until the maximum number of iteration is reached.

(7) When the maximum number of iteration is reached, the latest best solution $x_{\text {best }}$ is considered as the optimal values for the gains of PID controller.

Finally, the optimal PID gains through CS optimization are obtained as $k_{p}=1125.16, k_{i}=299.54$, $k_{d}=1448.35$.

\section{Simulation and Analysis}

With the optimal PID gains obtained in last section, the performance of the proposed CS-PID controller can be verified with a computer simulation in MATLAB/Simulink. In order to demonstrate the reliability and efficiency of the proposed CS-PID controller in the SAS, passive suspension is introduced as an intuitive comparative case. Also, the conventional PID controller is utilized for the same SAS system as another comparative case [9].

As aforesaid, this research aims to improve the ride comfort of the vehicle. So, the vertical acceleration of the sprung mass which can be used to quantify the ride comfort is taken as the evaluation index to assess the performance of the proposed system. The comparison of the vertical acceleration of the sprung mass using different control methods is shown in Fig. 3.

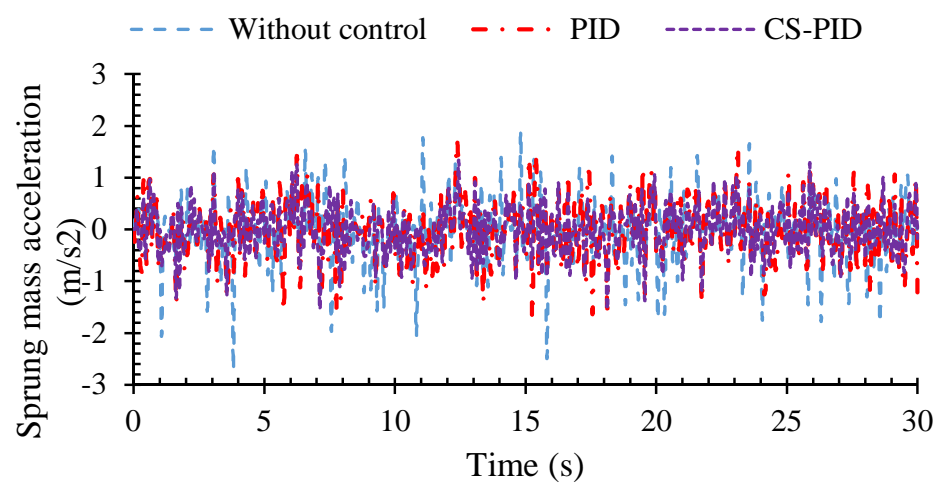

Fig. 3 Comparison of vertical acceleration of sprung mass.

In Fig. 3, the item without control stands for passive suspension system, PID means the SAS system with PID and the CS-PID is the proposed SAS system. Regarding the ride comfort, a small sprung mass acceleration represents a good ride comfort. It can be seen from Fig. 3 that the passive suspension system performs the worst while the SAS with the CS-PID strategy achieves the best ride comfort.

Table 2. Comparison of RMS values and peak values.

\begin{tabular}{ccc}
\hline Schemes & RMS value & Peak value \\
\hline Passive & 0.3436 & 2.7136 \\
Conventional PID & 0.3257 & 1.7075 \\
CS-PID & $\mathbf{0 . 2 3 4 3}$ & $\mathbf{1 . 5 4 8 3}$ \\
\hline
\end{tabular}

To examine the performance of the proposed method more in-depth, a root mean square (RMS) value and a peak value of the vertical acceleration of sprung mass are calculated for different control schemes. Table 2 shows the RMS values and peak values of different control schemes. For both the RMS value and the peak value, the smaller the value, the better the performance is. The CS-PID scheme keeps the smallest RMS value and the peak value compared with the other schemes, 
as described in Table 2. In a word, the simulation result shows that proposed CS-PID strategy is effective in improving the ride comfort of the vehicle.

\section{Conclusions}

A CS-PID strategy for the damping force control is proposed in this work in order to improve the ride comfort of the vehicle for different driving conditions. The quarter car dynamic model with an air spring and an adjustable damper has been successfully developed. The proposed CS-PID strategy has been well designed and applied to the SAS system. The simulation results show that the CS-PID strategy is effective in improving the ride comfort of the vehicle. It is believed that this work will provide guidance for the improvement of vehicle ride comfort.

\section{Acknowledgments}

This work was supported by the Science and Technology Development Fund of Macau S.A.R [Grant No: 107/2013/A3]. The authors would also like to thank Mr. Chan Kim Nam.

\section{References}

[1] H. Chen, C. Long, C.-C. Yuan, H.-B. Jiang, Non-linear modelling and control of semi-active suspensions with variable damping, Veh. Syst. Dynam. 51(10) (2013) 1568-1587.

[2] C. Poussot-Vassal, O. Sename, L. Dugard, R. Ramirez-Mendoza, L. Flores, Optimal skyhook control for semi-active suspensions, Mechatron. Syst. 39(16) (2006) 608-631.

[3] I. Mihai, F. Andronic, Behavior of a semi-active suspension system versus a passive suspension system on an uneven road surface, Mech. 20(1) (2014) 64-69.

[4] V. Sankaranarayanan, M. Emekli, B. Gilvenc, L. Guvenc, E. Ozturk, Ş. Ersolmaz, et al., Semiactive suspension control of a light commercial vehicle, Mechatron. IEEE/ASME Trans. 13 (2008) 598-604.

[5] H. Metered, P. Bonello, S. Oyadiji, An investigation into the use of neural networks for the semi-active control of a magnetorheologically damped vehicle suspension, Proc. Inst. Mech. Eng. Part D, J. Automobile Eng. 224(7) (2010) 829-848.

[6] P. Dash, L. C. Saikia, N. Sinha, Comparison of performances of several Cuckoo search algorithm based 2DOF controllers in AGC of multi-area thermal system, Int. J. Electric. Power \& Energy Syst. 55 (2014) 429-436.

[7] P. K. Wong, K. I. Wong, C. M. Vong, C. S. Cheung, Modeling and optimization of biodiesel engine performance using kernel-based extreme learning machine and cuckoo search, Renew. Energy. 74 (2015) 640-647.

[8] P. K. Wong, Z. Xie, J. Zhao, T. Xu, F. He, Analysis of automotive rolling lobe air spring under alternative factors with finite element model, J. Mech. Sci. Technol. 28(12) (2014) 5069-5081.

[9] X. S. Yang, S. Deb, Cuckoo search: recent advances and applications, Neural Comput. Appl. 24(1) (2014) 169-174. 